\title{
Calidad educativa y gestión institucional en la Universidad Nacional Intercultural de la Amazonía, Perú
}

\author{
Educational quality and institutional management at the \\ National Intercultural University of the Amazon, Peru
}

Qualidade educacional e gestão institucional na

Universidade Intercultural Nacional da Amazônia, Peru

\author{
WALTER ARTURO QUISPE CUTIPA ${ }^{1}$ \\ LILIAM CAROLA ZEVALLOS SOLIS ${ }^{2}$ \\ JOSÉ LUIS SANGAMA SÁNCHEZ3
}

\begin{abstract}
RESUMEN
El objetivo principal de esta investigación es determinar la relación de la calidad educativa con la gestión institucional en el personal que labora en la Universidad Nacional Intercultural de la Amazonía, Pucallpa, 2018. Como hipótesis central, la calidad educativa tiene relación directa y significativa con la gestión institucional en la Universidad Nacional Intercultural de la Amazonía, Pucallpa, 2018. Para tal fin, se llevó a cabo un estudio descriptivo, de diseño no experimental, como método general el científico y como específicos, el descriptivo, el estadístico y el hipotético deductivo. La investigación fue aplicada, de nivel correlacional y diseño descriptivo-correlacional; la cual empleó la técnica de la encuesta y como instrumentos los cuestionarios de encuesta para determinar los niveles de relación entre dichas variables de estudio. Los resultados fueron analizados con el programa estadístico SPSS v.22. Como resultado de esta investigación, se ha concluido con un nivel de significancia del 5\% que la calidad educativa tiene relación directa y significativa con la gestión institucional en la Universidad Nacional Intercultural de la Amazonía, Pucallpa, 2018, de acuerdo a los resultados se evidencia una calidad educativa media tendiendo a buena, constituyéndose en un factor determinante hacia la gestión institucional eficaz, lo cual se refleja en una percepción positiva de la Universidad Nacional Intercultural de la Amazonía por parte de la comunidad universitaria.
\end{abstract}

Palabras clave: Acreditación; evaluación; calidad; ética; investigación. 


\begin{abstract}
The main objective of this research is to determine the relationship between educational quality and institutional management in the personnel who work at the National Intercultural University of the Amazon, Pucallpa, 2018. As a central hypothesis, educational quality has a direct and significant relationship with institutional management at the National Intercultural University of the Amazon, Pucallpa, 2018. To this end, a descriptive study of non-experimental design was carried out, as a general method was scientific and as specifics were the descriptive, the statistical and the hypothetical deductive. The research was applied, correlational level and descriptive-correlational design; which used the survey technique and as instruments the survey questionnaires to determine the levels of relationship between these study variables. The results were analyzed with the statistical program SPSS v.22. As a result of this research, it has been concluded with a significance level of 5\% that educational quality has a direct and significant relationship with institutional management at the National Intercultural University of the Amazon, Pucallpa, 2018. According to the results, an average educational quality tending to good is evidenced, becoming a determining factor towards effective institutional management, which is reflected in a positive perception of the National Intercultural University of the Amazon by the university community.
\end{abstract}

Keywords: Accreditation; evaluation; quality; ethics; research.

\title{
RESUMO
}

O principal objetivo desta pesquisa é determinar a relação entre qualidade educacional e gestão institucional no pessoal que trabalha na Universidade Intercultural Nacional da Amazônia, Pucallpa, 2018. Como hipótese central, a qualidade educacional tem uma relação direta e significativa com a gestão institucional da Universidade Intercultural Nacional da Amazônia, Pucallpa, 2018. Para tanto, foi realizado um estudo descritivo de delineamento não experimental, sendo o método geral o científico e específicas eram o descritivo, o estatístico e o hipotético dedutivo. A pesquisa foi aplicada, nível correlacional e delineamento descritivo-correlacional; que utilizou a técnica de pesquisa e como instrumentos os questionários de pesquisa para determinar os níveis de relacionamento entre essas variáveis do estudo. Os resultados foram analisados com o programa estatístico SPSS v.22. Como resultado desta pesquisa, concluiu-se com um nível de significância de 5\% que a qualidade educacional tem uma relação direta e significativa com o gerenciamento institucional da Universidade Intercultural Nacional da Amazônia, Pucallpa, 2018. De acordo com os resultados, evidencia-se uma qualidade educacional média que tende a ser boa, tornando-se um fator determinante para uma gestão institucional eficaz, o que se reflete em uma percepção positiva da Universidade Intercultural Nacional da Amazônia pela comunidade universitária.

Palavras-chave: Acreditação; avaliação; qualidade; ética; investigação. 


\section{INTRODUCCIÓN}

Jofré (2013) sostiene que la calidad en la educación asegura a todos los jóvenes la adquisición de los conocimientos, capacidades, destrezas y actitudes necesarias para la vida adulta. De esta definición se puede inferir que:

- La calidad educativa es básica para la adquisición de conocimientos.

- La calidad del entorno depende de la adquisición de capacidades, destrezas y actitudes.

- Contribuye al proceso de formación integral de los estudiantes.

- Contribuye con el desarrollo de competencias y capacidades

- Demanda un compromiso de cada miembro de la comunidad con sus roles y tareas.

- Permite el aprendizaje y perfeccionamiento permanente.

- Demanda de una práctica permanente de evaluación y retroalimentación

Pérez (2008) refiere que la educación es de calidad cuando está dirigida a satisfacer las aspiraciones del conjunto de sectores integrantes de la sociedad a la que está dirigida; si, al hacerlo, se alcanzan efectivamente las metas que en cada caso se persigue. La calidad educativa consiste en hacer bien el trabajo desde el principio, responder a las necesidades de los usuarios, administrar óptimamente, actuar con coherencia, un proceso o modo de hacer; satisfacer al cliente/usuario; disfrutar con el trabajo y ofrecer lo mejor de uno mismo; reducir costes inútiles, evitar fallos; ser más eficaz, eficiente, productivo.

En suma, debemos entender por calidad educativa la mejora continua, a través del cumplimiento de criterios de eficacia y eficiencia, pertinencia y equidad; es decir, al conjunto de actividades encaminadas a la satisfacción de las necesidades y consecución de metas que produce efectos positivos en los protagonistas del proceso educativo: estudiantes y docentes.

Respecto a las dimensiones, según Pozner (2000) la existencia de algunas categorías o dimensiones existentes de calidad educativa son: ${ }^{3}$

Eficacia: Del latín efficač́a, la eficacia es la capacidad de alcanzar el efecto que espera o se desea tras la realización de una acción. No debe confundirse este concepto con el de eficiencia (del latín efficientĭa), que se refiere al uso racional de los medios para alcanzar un objetivo predeterminado (es decir, cumplir un objetivo con el mínimo de recursos disponibles y tiempo.

Pertinencia: es la cualidad de pertinencia. Se trata de un adjetivo que hace mención de lo perteneciente o correspondiente a algo o a aquello que tiene a propósito. "La propuesta demostró su pertinencia al solucionar uno de los principales problemas de la empresa". Existen distintos acercamientos a la noción de pertinencia. La pertinencia de la educación está vinculada al lugar que ocupa la formación en la sociedad. Dado que la educación básica se considera como un derecho humano, el debate gira en torno a la pertinencia de la educación superior en un contexto social: qué conocimientos difundir, con qué objetivo y cómo modificar la realidad a partir de la formación. 
Procesos: La noción de proceso halla su raíz en el término de origen latino processus. Según informa el Diccionario de la Real Academia Española, este concepto describe la acción de avanzar o ir para adelante, al paso del tiempo y al conjunto de etapas sucesivas advertidas en un fenómeno natural o necesario para concretar una operación artificial.

Respecto a la segunda variable, Gestión Institucional, para Chenet (2017) este intentar predecir la eficacia de la gestión institucional en los diferentes contextos en especial en el sistema universitario donde la mayoría de los docentes tienen aula a cargo y al mismo tiempo cargos administrativos lo que dificulta la óptima gestión institucional.

La gestión universitaria para la transformación de la Universidad, según Ortega (2006) menciona que, quien aborda el proceso a ser llevado adelante por el gerente educativo para lograr la transformación de la institución que dirige a fin de que pueda ofrecer un servicio de excelencia. Así, analiza el perfil que dicho gerente debe tener, el proceso de dirección a ejercer y el conjunto de competencias requeridas para ello como, liderazgo, toma de decisiones y generación de un clima de participación y trabajo en equipo en la escuela, junto al instrumental metodológico requerido para lograrlo.

Ser directivo o integrante del equipo directivo de una entidad educativa universitaria, es una oportunidad para poder llevar adelante la gestión de esa institución, cuyo objeto social es formar profesionales para contribuir a su desarrollo como personas y como ciudadanos. En ese campo hay muchos elementos (acciones y prácticas) que coexisten, interactúan y se articulan entre sí de manera dinámica, por lo que influyen unos a otros. Esos elementos se pueden distinguir y clasificar según la naturaleza de las acciones porque hay elementos de índole pedagógica, otros de carácter administrativo y organizativo y otros de tipo social comunitario. Esa distinción permite observar que al interior de la organización y de sus procesos de gestión existen dimensiones o planos de acciones diferentes, pero complementarios de la acción de los profesores y del nivel directivo.

A pesar del rechazo que a veces manifiestan los propios directivos y el profesorado en general a asumir el rol del liderazgo en la educación, casi toda la literatura sobre el tema subraya la importancia que el ejercicio de un cierto liderazgo institucional tiene para la calidad de la educación. Para Cáceres (2007) la gestión de la calidad total reposa sobre lo que desde una perspectiva jerárquica constituye su vértice superior, esto es, la dirección. Se confirma que el rector, tanto en su perfil profesional como técnico, experto tanto en organización, como en su rol de líder institucional, es un factor determinante de la calidad educativa.

El objetivo general de este trabajo fue determinar la relación de la calidad educativa con la gestión institucional en el personal que labora en la Universidad Nacional Intercultural de la Amazonía, Pucallpa, 2018. Mientras que los objetivos específicos fueron determinar la relación de la calidad educativa con la organización en el personal que labora en la Universidad Nacional Intercultural de la Amazonía, Pucallpa, 2018; determinar la relación de la calidad educativa con la estructura en el personal que labora en la Universidad Nacional Intercultural de la Amazonía, Pucallpa, 2018; determinar la relación de la calidad educativa con las relaciones personales en el personal que labora en la Universidad Nacional Intercultural de la Amazonía, Pucallpa, 2018; y determinar la relación de la calidad educativa con la normatividad en el personal que labora en la Universidad Nacional Intercultural de la Amazonía, Pucallpa, 2018. 


\section{METODOLOGÍA}

En esta investigación se utilizó el método científico y como métodos específicos el método descriptivo, el estadístico y el hipotético deductivo (Kerlinger \& Lee, 2002). Es de tipo aplicada, de nivel correlacional (Oseda, 2018). Asimismo, presenta un diseño descriptivo correlacional que se observa en el siguiente esquema:

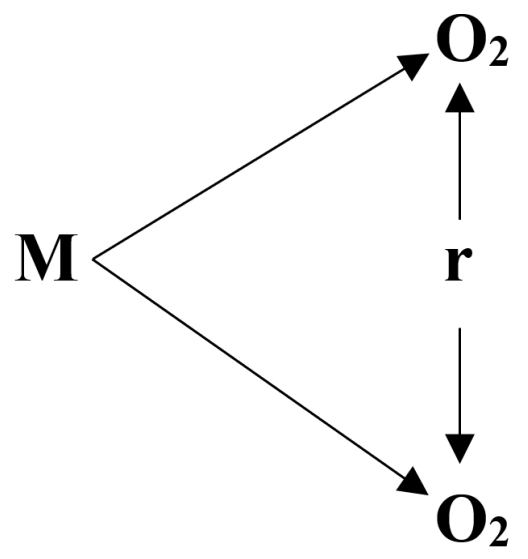

Donde:

- $\mathrm{M}=$ Muestra

- $\mathrm{O}_{1}=$ Observación de la variable 1 .

- $\mathrm{O}_{2}=$ Observación de la variable 2 .

- $\quad$ rho $=$ Correlación entre dichas variables.

La población estuvo conformada por 140 docentes entre ordinarios y contratados de la Universidad Nacional Intercultural de la Amazonía, Pucallpa en el periodo 2018-II. Muestreo: probabilístico y estuvo conformado por 53 docentes. Factores de exclusión: Varios docentes se reusaron ser encuestados por cuestiones particulares. Técnicas de recojo de información: Se consideró a la encuesta con su instrumento el cuestionario el cual se aplicó para las dos variables de estudio. La confiabilidad se hizo por el método de mitades partidas $\mathrm{Cxy}=0.952$ y la validez por el juicio de experto, alcanzado este el $95 \%$ en dicho criterio.

\section{RESULTADOS}

Tabla 1.

Niveles de la Variable 1: Calidad Educativa

\begin{tabular}{ccc} 
Niveles & Frecuencia & Porcentaje \\
\hline Muy buena & 7 & 13.21 \\
Buena & 42 & 79.25 \\
Media & 4 & 7.55 \\
Deficiente & 0 & 0.00 \\
\hline Total & $\mathbf{5 3}$ & $\mathbf{1 0 0 . 0 0}$ \\
\hline
\end{tabular}

Fuente: Base de datos de los investigadores 


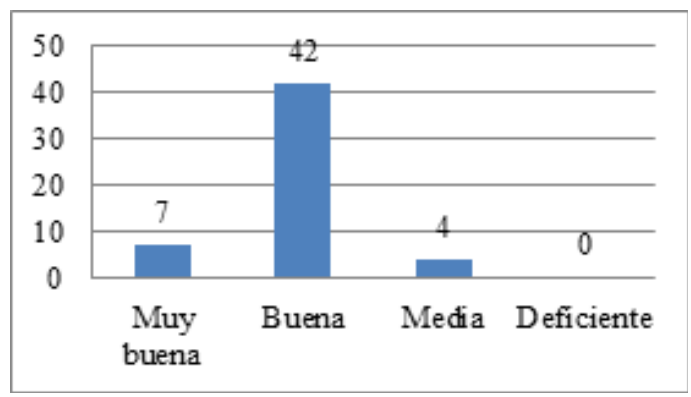

Figura 1. Niveles de la Variable 1: Calidad Educativa

Fuente: Base de datos de los investigadores

Según la tabla y figura 1, con respecto a la variable Calidad Educativa, se aprecia que el $79.25 \%$ de los docentes encuestados sostienen que la calidad educativa de la universidad es buena, el $13.21 \%$ sostienen que la calidad es muy buena, mientras que el $7.55 \%$ de los encuestados sostienen que el nivel es medio o regular.

Ahora, mostramos el consolidado de la variable "Gestión Institucional”, el cual se resume en la siguiente tabla y figura:

Tabla 2.

Niveles de la Variable 2: Gestión Institucional

\begin{tabular}{ccc} 
Niveles & Frecuencia & Porcentaje \\
\hline Muy buena & 0 & 0.00 \\
Buena & 2 & 3.77 \\
Media & 39 & 73.58 \\
Deficiente & 12 & 22.64 \\
\hline Total & $\mathbf{5 3}$ & $\mathbf{1 0 0 . 0 0}$ \\
\hline
\end{tabular}

Fuente: Base de datos de los investigadores

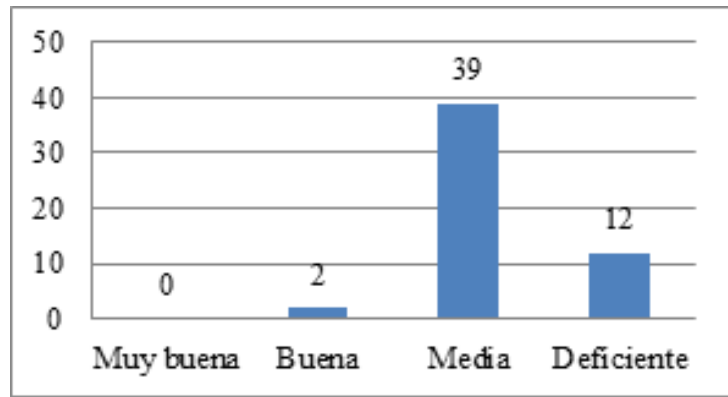

Figura 2. Niveles de la Variable 2: Gestión Institucional

Fuente: Base de datos de los investigadores 
Según la tabla y figura 2, respecto a la variable Gestión Institucional, se observa que el $73.58 \%$ sostiene que la gestión institucional en la universidad es medio o regular, luego $22.64 \%$ sostienen que el nivel es muy deficiente y el 3.77\% sostienen que el nivel es bueno de la Gestión Institucional en la universidad. Aquí se observa que el desempeño de nuestras autoridades no es del todo adecuado; claro reflejo de los que sucede en las universidades públicas del país.

Tabla 3.

Coeficiente de correlación

\begin{tabular}{|rl|r|r|}
\hline & Calidad Educativa & Gestión institucional \\
\hline \multirow{3}{*}{ Calidad Educativa } & Spearmann Correlation & 1 &, 842 \\
\cline { 2 - 4 } & Sig. (2-tailed) & &, 000 \\
\cline { 2 - 4 } & $\mathrm{N}$ & 53 & 53 \\
\hline Gestión institucional Spearmann Correlation &, 842 & 1 \\
\hline Sig. (2-tailed) &, 000 & 53 \\
\hline $\mathrm{N}$ & 53 & \\
\hline
\end{tabular}

Significatividad al $1 \% * *$

Respecto a la prueba de hipótesis, como el diseño utilizado fue el descriptivo-correlacional, el estadígrafo utilizado fue el coeficiente de correlación de Spearman, prueba bilateral a dos colas. Ahora bien, como la rho $=0,842$ y la $t$ calculada $\left(t_{c}\right)$ con los datos procesados es igual a 11,15 y este cae en la zona de rechazo; entonces se rechaza la $\mathrm{H}_{0}$, y se acepta la hipótesis alterna $\mathrm{H}_{1}$; que dice: Se concluye que existe una correlación directa y significativa entre la calidad educativa con la gestión institucional en la Universidad Nacional Intercultural de la Amazonía, Pucallpa, 2018.

\section{DISCUSIÓN}

Los resultados obtenidos evidencian una correlación directa y significativa entre la calidad educativa y la gestión institucional en la Universidad Nacional Intercultural de la Amazonía, Pucallpa, 2018; es decir a una mejor calidad educativa, también le corresponde una buena gestión institucional y viceversa, el cual es muy favorable para esta universidad pública. Asimismo, la calidad educativa tiene una correlación lineal significativa con la gestión institucional en la Universidad Nacional Intercultural de la Amazonía, Pucallpa, 2018, ya que se obtuvo una rho $=0,842$. De acuerdo con la interpretación de Hernández, Fernández y Baptista (2010), el "rho" obtenido se ubica en una correlación positiva considerable, lo cual nos permite señalar que, a mayor eficiencia de la calidad educativa, mayor será el nivel de la gestión institucional o viceversa.

Este resultado indica que la eficiencia en el servicio académico brindado, así como un óptimo manejo de los recursos de la Universidad; permite una adecuada planeación estratégica, una buena percepción de cultura organizacional y una dirección eficiente de la universidad. 
Sobre la hipótesis específica $\mathrm{N}^{0} 01$, la calidad educativa tiene relación directa y significativa con la organización de la Universidad Nacional Intercultural de la Amazonía, Pucallpa, 2018; se infiere que existe una relación directa moderada entre los procesos administrativos organizativos, tales como, registro de datos, de notas, expedición de certificados y actas; así como el diverso trámite documentario que se realiza en la universidad con la gestión institucional del planeamiento estratégico, organización y dirección ejecutada.

Sobre la hipótesis específica $\mathrm{N}^{\mathrm{o}} 02$, la calidad educativa tiene relación directa y significativa con la estructura en la Universidad Nacional Intercultural de la Amazonía, Pucallpa, 2018. Se deduce que hay una relación moderada entre la estructura organizativa, la capacitación de personal; aplicación de la normatividad en cuanto a sus funciones y deberes, como también en los derechos que se le asiste; con la gestión de la organización y planeación estratégica de la universidad.

Sobre la hipótesis específica $\mathrm{N}^{\mathrm{o}} 03$, la calidad educativa tiene relación directa y significativa con las relaciones interpersonales en la Universidad Nacional Intercultural de la Amazonía, Pucallpa, 2018. Se infiere que las relaciones personales e interpersonales es crucial para tener una buena gestión institucional. Al respecto Bustamante (2007) menciona que el manejo y distribución de los recursos humanos, es en función a las buenas relaciones interpersonales dentro de las organizaciones educativas.

Sobre la hipótesis específica $\mathrm{N}^{\mathrm{0}} 04$, la calidad educativa tiene relación directa y significativa con la normatividad en la Universidad Nacional Intercultural de la Amazonía, Pucallpa, 2018, se deduce que el proceso de captación de recursos propios, elaboración del presupuesto, la contabilidad, la presentación de los informes económicos referidos a la normatividad administrativa vigente tiene una relación positiva muy fuerte con la gestión institucional, sobre todo, en el campo de percepción de la cultura organizacional y la toma de decisiones en el campo de la dirección.

Como teoría sustentadora de la variable gestión institucional hemos tomado a Alvarado (2009) que menciona que en las instituciones públicas del sistema educativo peruano, la gestión imperante es la regular o media, y precisamente en esa medida los resultados estadísticos de la tesis manifiestan algo similar, existiendo una tendencia, ya que la calidad educativa según la percepción de los trabajadores de la Universidad Nacional Intercultural de la Amazonía sea buena, pero la gestión Institucional, sustentada en Ayala (2015) que se basa en gran medida al liderazgo del rector (Presidente de la Comisión Organizadora) de la Universidad es media o regular. En esa óptica, los resultados estadísticos de la investigación indican que la gestión del rector no es del todo satisfactoria, sin embargo, se aprecia una relación directa fuerte y significativa entre la gestión institucional y la calidad educativa en la mencionada universidad, con lo cual demostramos nuestra hipótesis de investigación en todo su sentido y significado.

\section{CONCLUSIONES}

Se ha determinado que la calidad educativa tiene relación directa y significativa con la gestión institucional en la Universidad Nacional Intercultural de la Amazonía, Pucallpa, 2018; ya que de acuerdo con los resultados se evidencia una calidad educativa buena, es un factor determinante que permite una gestión institucional eficaz, lo cual se refleja en una percepción positiva de la universidad por parte de la comunidad educativa universitaria. 
La calidad educativa tiene relación directa y significativa con la organización en la Universidad Nacional Intercultural de la Amazonía, Pucallpa, 2018, ya que, al brindar un buen servicio académico, así como también los registros, la estadística, los procesos administrativos, servicios educativos y trámites documentarios, esta afecta el proceso de la gestión institucional de manera directa.

La calidad educativa tiene relación directa y significativa con la estructura en la Universidad Nacional Intercultural de la Amazonía, Pucallpa, 2018, ya que se evidencia la falta de actualización respecto a la estructura organizativa; esto lógicamente determina la gestión institucional, principalmente en el campo de la cultura organizacional.

La calidad educativa tiene relación directa y significativa con las relaciones interpersonales en la Universidad Nacional Intercultural de la Amazonía, Pucallpa, 2018; donde las relaciones interpersonales no son tan buenas, por lo tanto, la motivación laboral es regular, afectando el ambiente laboral el cual repercute en la gestión institucional.

La calidad educativa tiene relación directa y significativa con la normatividad en la Universidad Nacional Intercultural de la Amazonía, Pucallpa, 2018, ya que la gestión de recursos materiales basado en la normatividad vigente afecta el proceso de rendimiento laboral y el cumplimiento de los objetivos institucionales establecidos en el Proyecto Educativo Institucional, en otras palabras, no ayuda a la consecución de las metas de la planeación estratégica.

\section{REFERENCIAS}

Alvarado, O. (2009). Gestión Educativa. Enfoques y Procesos. Universidad de Lima Fondo de Desarrollo Editorial.

Ayala, G. (2015). Nuevas propuestas para la gestión educativa. Biblioteca para la Actualización del Maestro.

Bustamante, D. (2007). Comportamiento organizacional (10 a edición). Thomson.

Cáceres, P. (2007). Administración Estratégica de Calidad. Ariel.

Chenet, M. (2017). Administración Gerencial y Estratégica. USIL.

Hernández, R., Fernández, C. \& Baptista, P. (2010). Metodología de la investigación. Mc Graw Hill.

Jofré, A. (2013). Enfoques gerenciales modernos. Ediciones Delphi Libre de Costa Rica.

Kerlinger, F., \& Lee, H. (2002). Investigación del comportamiento. Interamericana S.A.

Ortega, M. (2006). Gestión Educativa. McGraw-Hill.

Oseda, D. (2018). Fundamentos de investigación cientifica. Soluciones Gráficas.

Pérez, P. (2008). Toma de decisiones en el Sistema Educativo de Venezuela. Tesis.

Pozner, P. (2000). Gestión Educativa Estratégica. IIPE - UNESCO. 